\title{
-Communication-
}

\section{Fabrication and Evaluation of PFC (Plastic Formed Carbon) Electrodes for Voltammetric Use}

\author{
Hiroko KANEKO*, Akira NEGISHI, Yoshihisa SUDA $^{\dagger}$ and Takamasa KAWAKUBO ${ }^{\dagger}$ \\ Received February 12, 1993 ; Accepted March 16, 1993
}

\section{INTRODUCTION}

We found that mechanical pencil leads named graphite reinforcement carbon(GRC) can be used as voltammetric electrodes ${ }^{1)}$. Although the GRC electrodes showed good performance and characteristics for electrochemical measurements ${ }^{2-4}$, they can not be used in non-aqueous solutions because of containing mineral oils ${ }^{5}$ ). Therefore, we tried to develop new carbon electrodes impermeable by solvents with which show suppressed back ground currents in organic solvents. Various carbon electrodes named PFC(Plastic Formed Carbon) have been made of highly purified carbon. They have been manufactured from mixtures of graphite powder and organic binder like furan resin which become impermeable amorphous carbon after carbonizing at $1000^{\circ} \mathrm{C}$ under inert gas atmosphere $^{6)}$.

In order to use the $\mathrm{PFC}$ as convenient electrode materials, it is necessary to show the electrochemical characteristics and the performance of the electrodes. In the present paper, fabrication concept of the electrode has been introduced and electrochemical behavior of the PFC electrode has been discussed by comparing with the surface of HOPG(highly oriented pyrolytic graphite).

\section{CONCEPT OF PFC EIECTRODES}

The conceptual map of the PFC is shown in Fig.1. The horizontal axis shows graphite contents mixed with organic binder, and the vertical axis shows diameter of the PFC rods. Many geometrical PFC rods with various graphite contents are involved in this map.

Electrotechnical Laboratory, 1-1-4 Umezono, Tsukuba, Ibaraki 305 JAPAN

tMitsubishi Pencil Co.Ltd., 1091 Hagiyadoura Tatsuishi, Fujioka, Gumma $37 \dot{5}$ JAPAN

Key words: carbon electrode, plastic formed carbon, PFC, HOPG, highly oriented pyrolytic graphite, cyclic voltammetry
The mechanical pencil lead, GRC, is a sort of the PFC with much contents of natural graphite. It is located an upper right part of the map. On the map, the glassy carbon is located on the left line of the map and carbon fibers lie in the lower part of the map, because there are many type of carbon fibers with different contents of graphite.

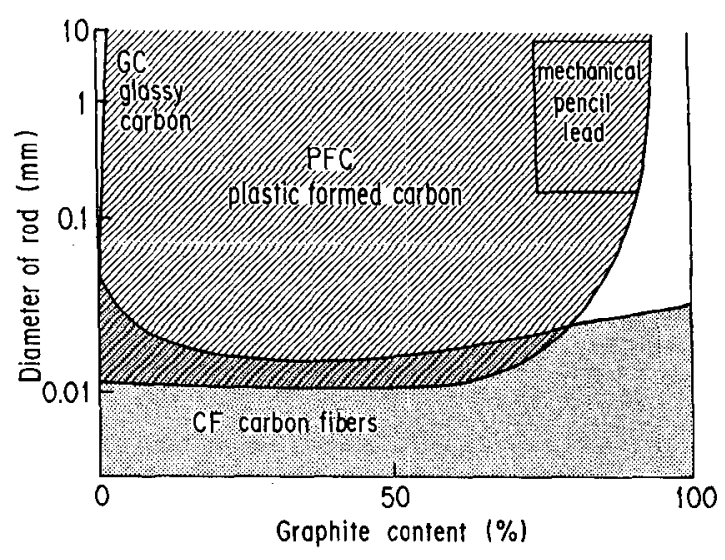

Fig.1 Conceptual map of plastic formed carbon

\section{EXPERIMENTAL.}

Raw materials of the PFC used here were $50 \%$ natural graphite powder of about $1 \mu \mathrm{m}$ size and $50 \%$ plastic binder. They were kneaded well after dispersing the mixture, and extruded through many size of thin dice. This was the precursor of the PFC rod. After drying and oxidizing it at about 100 to $300^{\circ} \mathrm{C}$ in air, the precursor was carbonized at $1000^{\circ} \mathrm{C}$ in an inert gas atmosphere.

The PFC disk electrodes of $0.5 \mathrm{~mm}$ in diameter were used in this experiments. The side surface of the PFC electrodes were insulated with silicorn resin except for 5 mm from the end. For evaluation of the performance of the $\mathrm{PFC}$ electrode, $0.005 \mathrm{M}\left(\right.$ mole $\left.\mathrm{dm}^{-3}\right)$ ferro/ferri- 
cyanide ions in $1 \mathrm{M}$ potassium chloride solution were used. The basal and edge planes of the HOPG were used as comparable electrodes. The HOPG was so convenient that the surface could be renewed reproducibly before each experiment. When the basal plane was used as an electrode, the other plane of the HOPG was insulated with the silicorn resin.

\section{RESULTS AND DISCUSSION}

We have found that the side and the end surfaces of the PFC rod show different electrochemical behavior caused by molecular structure of carbon and the fabrication method[6]. In order to evaluate more clearly the electrochemical behavior of the PFC rod as an electrode, we compared back ground currents and redox reaction of ferro/ferricyanide ions at the PFC electrode with those at the HOPG electrode.

A back ground current at the a disk plane of the PFC electrode is shown in Fig. 2. Differential capacities at $O \mathrm{~V}$ vs. SCE were 50 $110 \mu \mathrm{F} \mathrm{cm}^{-2}$ at the PFC disk electrodes. These values were about $2-4$ times larger than the current at a dropping mercury electrode and nearly equal to double layer capacitance at polished edge plane of the HOPG electrode measured by $\mathrm{AC}$ impedance technique ${ }^{7)}$. Differential capacities at $0 \mathrm{~V}$ vs. SCE of back ground currents on side planes of the PFC electrodes were about $10 \mu \mathrm{F} \mathrm{cm}^{-2}$ 7). These values were almost the same as those at the basal plane of the HOPG electrode.

Voltammetric behavior of the PFC electrode is shown in Fig.3. The C.V.curves of the redox reaction of ferro/ferricyanide ions on the side and the disk plane of the PFC electrode have a similar pattern to those of the basal and the edge planes of the HOPG electrode. The current densities of the electrode reactions at the side plane and at the disk plane of the PFC electrode were about 0.5 and $3 \mathrm{~mA} \mathrm{~cm} \mathrm{~cm}^{-2}$, respectively.

Comparison in the C.V. curves and the differential capacity between the PFC electrode and the HOPG electrode suggests that graphite crystal edges are oriented on the surface of the disk plane of the PFC electrode. The disk plane of the PFC electrode is very active to ionic species as like the edge plane of the HOPG electrode. So, We can easily expose a new active surface by only cutting the PFC rods.
PFC 39

$1 \mathrm{MKCl}$

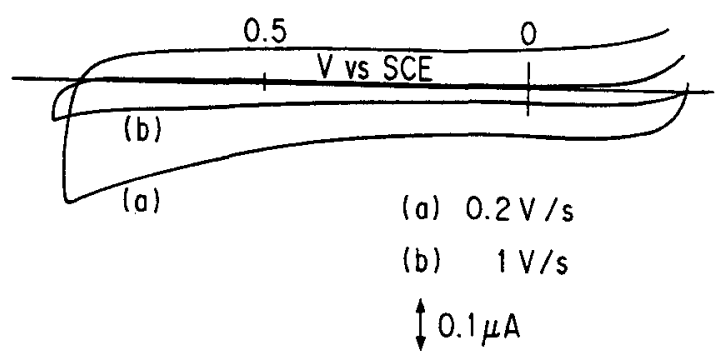

Fig.2 A back ground current on the PFC disk electrode
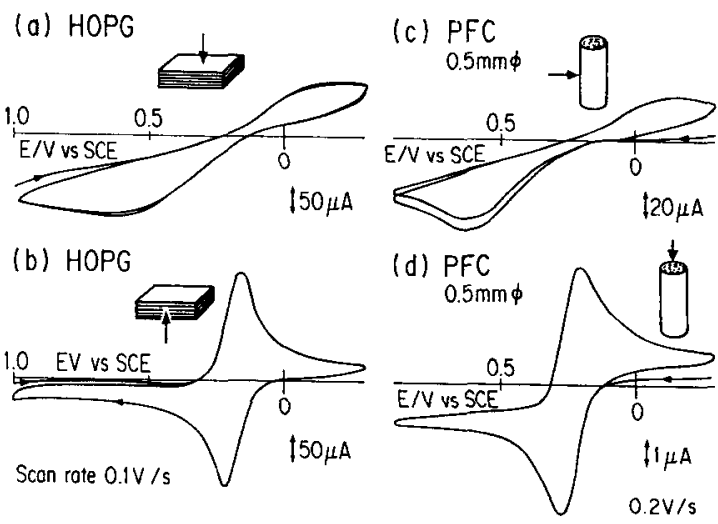

Fig.3 Cyclic voltammograms(C.V.) of ferro/ ferricyanide ion in $1 \mathrm{M}$ potassium chloride solution on the PFC electrodes and the HOPG electrods. (a) was a C.V.curve on a basal plane of the HOPG electrode and (c) was obtained on a side plane of the $\mathrm{PFC}$ electrode. (b) was the curve on an edge plane of the HOPG and (d) was obtained on a disk plane of the PFC electrode.

\section{REFERENCES}

1) H.Kaneko and K. Nozaki, Rev. Polarogr., 3355 (1987)

2) K.Aoki, T.Okamoto, H.Kaneko, K.Nozaki, and A.Negishi, J. Electroanal. Chem., 263 323331 (1989)

3) K.Aoki, A.Kobayashi, and N.Kato, Electroanalysis, 2, 31 (1990)

4) H.Kaneko, M.Yamada, and K.Aoki, Anal.Sci.6 439-442 (1990)

5) in preparation

6) T.Kawakubo, Y.Suda, H.Kaneko, A.Negishi, and M.Yamada, TANSO 1992 106-114

7) K.Kinoshita, "Carbon", John Wiley \& Sons, p.294 (1988) 